\title{
Exploring the use of image processing to survey and quantitatively assess historic buildings
}

\author{
C. González Manich, T. Kelman, F. Coutts, B. Qiu, \\ P. Murray, C. González-Longo \& S. Marshall \\ University of Strathclyde, Glasgow, UK
}

\begin{abstract}
Before architectural conservation takes place, a survey is conducted to assess the condition of the building and estimate the cost of the work. For façades, scaffolding is erected so that experts can access the building's whole extent and gather data for analysis. This paper presents the results of a collaborative and cross-disciplinary research project aiming to automate data capture and analysis techniques for conservation of stone façades. Our research demonstrates the feasibility of a new methodology for the survey and assessment of historic buildings and will facilitate frequent surveys with minimal disruption to the general public in cities. The project has embedded architects' expert knowledge into intelligent algorithms for automatically analysing images of facades. The combination of technologies allows for an efficient data capture while minimising the requirement for manual data analysis as well as more accurate estimates of its cost.
\end{abstract}

\section{INTRODUCTION}

\subsection{Context and aim of the project}

This paper presents a study which assessed the feasibility of applying a new methodology for the survey and assessment of historic buildings based on applying state-of-the-art image processing. This has the potential to facilitate frequent surveys with minimal disruption to the general public in cities.

The conservation of Built Heritage is a high value market and there is the need to continuously survey, assess and monitor historic buildings and structures. However, this is not always possible using the current state-of-the-art manual data gathering and data analysis approaches which can be labour intensive and time consuming. This can result in insufficient maintenance and monitoring leading to major problems and causing the loss of the heritage's value.

Nevertheless, the latest developments in image processing make this a technology with great potential to address this challenge. The possibility of automating the data capture and processing opens new opportunities in fields such as architecture conservation as it can reduce the time and cost of inspections making them more accessible.
In this work, we use traditional cameras and hyperspectral imaging systems. Where conventional digital imaging devices capture light over one (greyscale) or three (red, green, blue) distinct portions of the electromagnetic spectrum, a hyperspectral imaging system acquires light at hundreds or even thousands of different spectral bands. Similar to the way in which a spectrometer produces a spectrum of the light captured at a single point, a hyperspectral system produces a spectrum for each pixel in the image, see Figure 1. This combination of spatial and spectral detail provides significantly more information about a scene. In recent years it has been used in a number of different applications such as classification of Chinese tea (Kelman et al. 2013) and beef quality assessment (Qiao et al. 2015) and a number of new signal processing techniques have been developed to efficiently process the data (Zabalza et al. 2014) (Zabalza et al. 2015) .

In this context, the Architectural Design \& Conservation Research Unit (ADCRU) and the Centre for Signal and Image Processing (CeSIP) from the University of Strathclyde (Glasgow, UK) started collaborating in the research project presented in this paper.

The aim of the work is to create a new methodology for the survey and assessment of historic buildings based on state-of-the-art image processing technolo- 
gies. First, image data related to the condition of a historic building is captured. Then, its analysis is automated using new and intelligent image processing software modules developed for this purpose. Finally, the resulting information can later be used to assess the building condition and design the most adequate intervention in every case.

In the end, the outputs of the research can provide a more accurate, time and cost effective solution for estimating the cost of architectural conservation and to carry out better assessments and monitoring of the condition of the existing buildings.
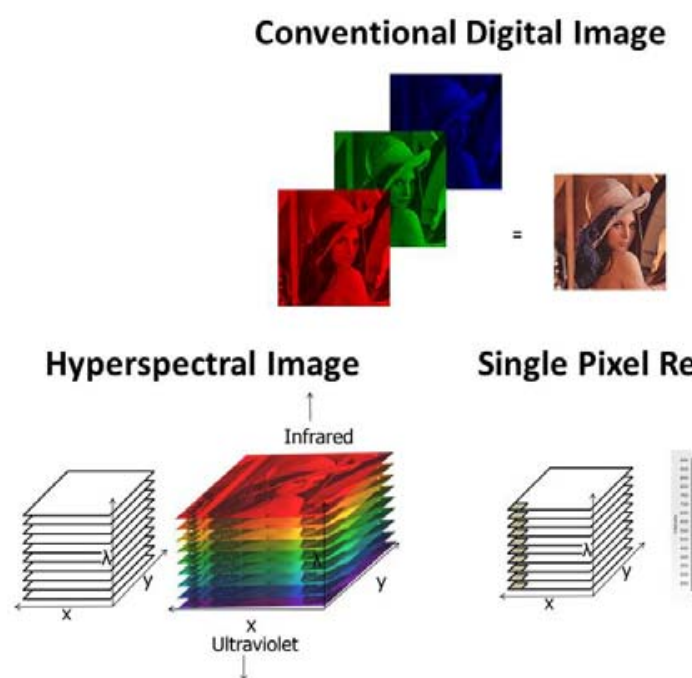

Single Pixel Representation

Figure 1: Conventional digital imaging and Hyperspectral imaging.

\subsection{Literature survey: precedents}

Image processing techniques have been implemented in a wide range of areas such as medicine (Dougherty 2009), automation (Bulanona et al. 2002), security (Sewaga et al. 2006) and defence (Colloquium 1990). In recent years, it has been adopted in the architecture field using a number of different devices and technologies as explained below.

One approach, (Lerma et al. 2000) used a digital colour camera integrated with a near infrared (NIR) camera. This allowed the capture of multispectral images of building façades as well as texture images generated from the four spectral regions - red, green, blue (RGB) and an NIR band. This data was found to be useful for recognising different kinds of limestone. This research also indicated that better classification of visually similar materials was achieved when using the NIR band rather than the RGB data. Later research (Lerma 2001) indicated that not only multispectral images but also multitemporal and multisensory images helped to increase classification accuracy. In further work, (Lerma 2005) different methodologies of classification were compared. A supervised maximum-likelihood classifier was considered as the best way to classify different wall materials. A minimum-distance classifier was quicker but had significantly lower accuracy.

In work presented by M.W. Hemmleb, (Hemmleb et al. 2005) a variety of illumination conditions were considered as a problematic source of error. Therefore, a new multispectral scanner was developed to apply active illumination which mapped different materials and corrosion (Hemmleb et al. 2006). However, this method was time consuming and it was concluded that further work was required to improve the robustness of the results.

Another trial (Palombi et al. 2008) was carried out with fluorescence LiDAR used on The Coliseum in Rome. The LiDAR systems used were not only able to distinguish different materials, but also able to differentiate stone masonry from different historic periods, which was helpful to identify previous conservation works that were not clearly recorded.

Thermal images show temperature fluctuations of material surfaces. As different materials have different specific heat capacities, the measured fluctuations can be used as a means of identification. Combining the thermal fluctuation data and the analysis of the RGB images, materials on a façade were classified using a maximum likelihood classifier (Sidiropoulou-Velidou et al. 2012).

Based on these precedents this paper aims to further develop the image processing techniques to allow automated analysis of stone masonry facades, identifying the different materials used as well as pathologies. This novel approach aims to improve the efficiency of the current manual survey process by using data from multiple sensors including traditional colour cameras and Hyperspectral Imaging systems.

\section{METHODOLOGY AND SCOPE}

In order to explore the possibility of applying stateof-the-art image processing to the challenge of surveying historic buildings, one case study was selected and surveyed using two different approaches.

The first approach follows the methodology of a traditional architectural conservation condition survey. It was carried out by ADCRU and was entirely 
based on professional expertise, involving visual inspection and manual work.

The second approach is based on image processing techniques. It was carried out by a team from CeSIP with the support of ADCRU. The objective in this case was to obtain RGB and hyperspectral images and process them to identify the mortar joints and classify the different pathologies.

In both cases, the scope of the survey only considered two types of information: the geometry of the masonry (stone units and mortar joints), and the pathologies.

The comparison of the results obtained in both approaches is used to evaluate the potential of this new proposed methodology.

\subsection{The case study}

The case study selected is a wall section $(371 \mathrm{~cm} \times$ $167 \mathrm{~cm}$ ) of the Glasgow Cathedral, located at the bottom right side of its main façade, as shown in Figure 2.

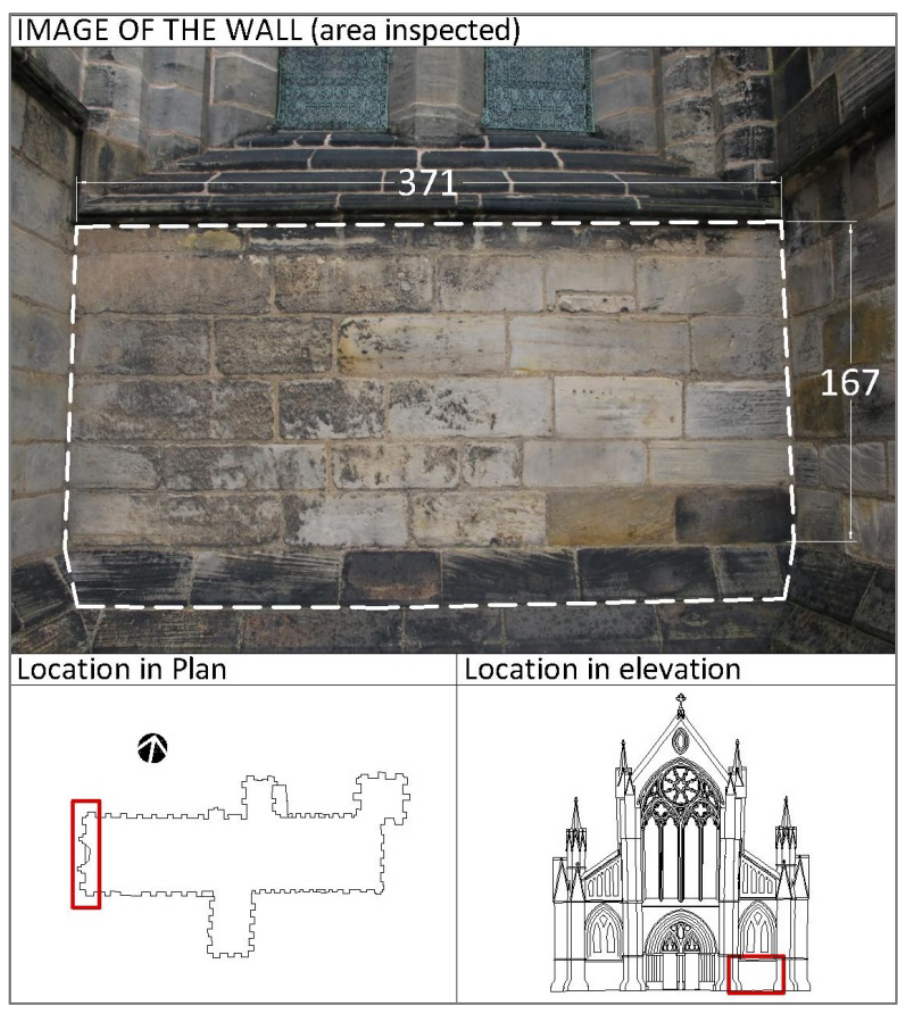

Figure 2: Image of the surveyed wall from the Glasgow Cathedral, including reference to its location in plan and elevation.
This area was chosen because the surface comprised of a regular ashlar masonry pattern simplifies the analysis and facilitates the comparison of the results from both studies. At the same time, it contains several different pathologies (and therefore colours and textures) making it an ideal sample for this study.

\subsection{Traditional survey}

Traditional surveys are mainly based on a manual process. Usually, an expert team gathers information about the geometry, history, construction details, materials and pathologies of a building. Every type of data is recorded separately in a set of plans and reports. Then, this layered information is combined and analysed to assess the condition of the building and propose the most adequate conservation. However, in this case, the scope of the work determined that it was only necessary to collect information about geometry and pathologies of the wall under study.

The data regarding geometry was provided by Historic Environment Scotland (HES) from a photogrammetry survey performed by one of their qualified teams. The resulting elevations contained the definition of the stone unit sizes and mortar joints thicknesses.

The condition survey was based on a visual inspection carried out by an experienced specialist conservation architect. Carefully examining the different stone units, she was able to identify the different pathologies present in the wall section, based on consistency and appearance of the surfaces and deposits - including texture and colour aspects - considering also the location and exposure of the individual stones and surfaces. This data was manually marked and annotated on a scaled elevation drawing using a graphic code based on the Italian Standard Normal 1/88 as shown in Figure 8.

\subsection{Image processing survey}

\subsubsection{Data Capture}

For the RGB capture, a Canon PowerShot G11 with Canon Zoom lens 5X15 was used. The images were captured at a resolution of $3648 \times 2736$ pixels.

Several hyperspectral images were also captured of the same wall. These were captured using a Hamamatsu ORCA-05G camera connected to a Specim V10E imaging spectrograph with a Schneider Compact VIS-NIR Lens. Using $4 \times 4$ binning, the images 
were captured at a resolution of $336 \times 1478$ spatial pixels, with each pixel containing 256 spectral bands ranging from $400 \mathrm{~nm}$ to $1000 \mathrm{~nm}$. Since this camera system is a line-scan system, either the object or the camera must be moved in order to construct an image of the scene. To achieve this, the system was mounted on a Zolix RAK100 motorised rotational stage attached to a tripod. See Figure 3.

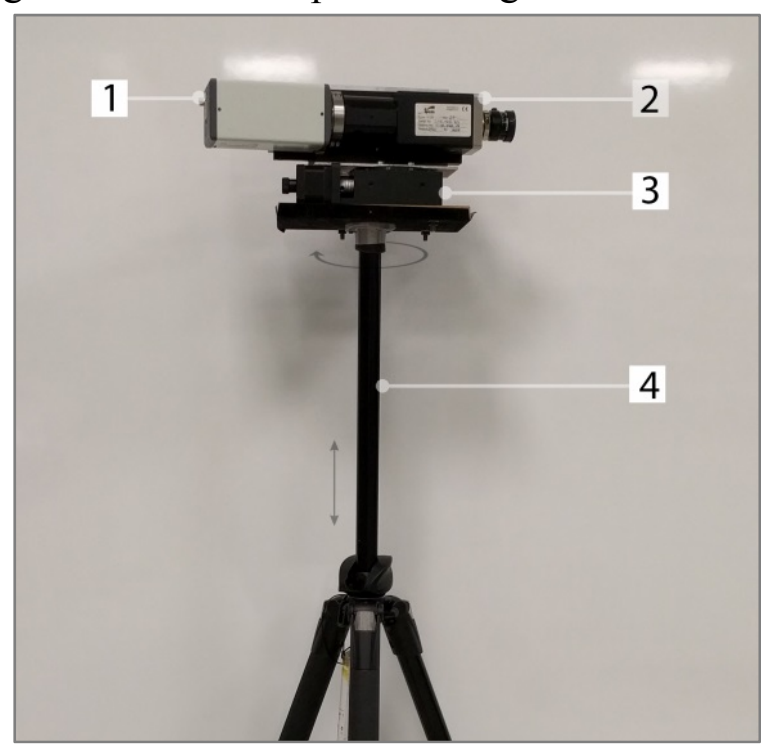

Figure 3: Hyperspectral camera used during the research work. 1) Camera 2) Spectrograph 3) Motorised Stage 4) Tripod.

The image was built up line by line as the system rotated. This rotation caused some distortion, as can be seen in Figure 4 where the horizontal lines have a slight curve to them. This can be corrected in future by translating the camera past the scene rather than rotating it or by registering points to the CAD model. The image shown in Figure 4 is the 100th spectral band in the data cube. To calibrate each band to a specific wavelength, a mercury calibration lamp with known spectral peaks was used. In this case, band 100 corresponds to $628 \mathrm{~nm}$.

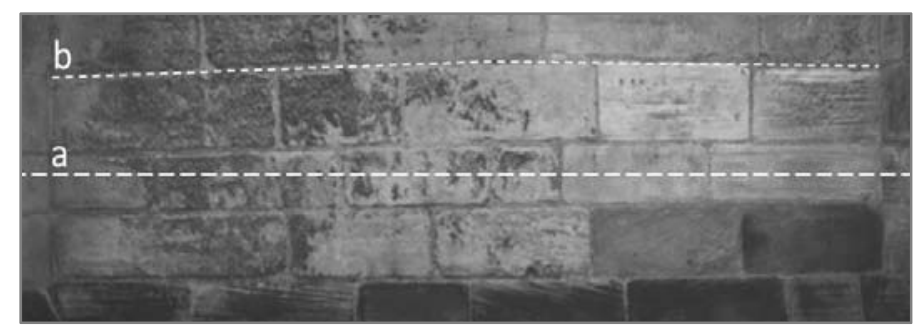

Figure 4: HSI_2563 part of the west facing wall at $682 \mathrm{~nm}$.

a) Horizontal reference line. b) Deformed line.

\subsubsection{Signal Processing Methodology}

\subsubsection{Mortar Detection}

A number of different signal/image processing techniques were used to detect the mortar regions of the image. The following algorithms were used: Law's texture analysis (Laws 1980) to extract feature vec- tors; Principal Component Analysis (PCA) (Jollie 2002) to reduce the dimensionality of the hyperspectral data; Support Vector Machines (SVM) (Cortes \& Vapnik 1995) to classify the texture features; 2-D filtering to enhance mortar regions; morphological openings, closings and top hats (Serra 1988) to remove noise; and marker controlled watershed segmentation (Beucher 1991) to segment the classification result.

Law's texture analysis uses 25 two-dimensional kernels obtained via the convolution of 5 onedimensional kernels with each other. The five onedimensional kernels are:

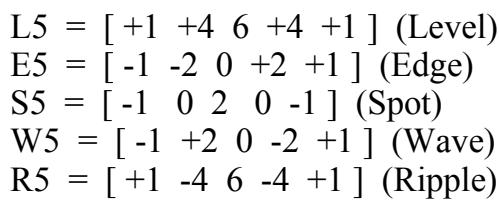

The 25 two-dimensional kernels produced are then convolved with each band in the input image. An RGB image has three bands, so after this technique has been applied each pixel has $25 \times 3=75$ features. The hyperspectral images have 256 bands. However some of these contain large amount of noise due to the properties of the spectrograph and the sensor.

The 200 useable bands would produce $25 \times 200=$ 5,000 features for each pixel. Rather than using these 5,000 features, PCA is employed to reduce the dimensionality of the hyperspectral data before texture analysis. PCA exploits the redundancy in multidimensional data and reorders it in such a way that most of the variance is contained within the first few components. The 200 band hyperspectral image can therefore be reduced to a 10 band PCA image with minimal loss of information. After texture analysis, this produces $25 \times 10=250$ features for each pixel.

As well as using the principal components from the hyperspectral image, three bands roughly corresponding to the response of the human eye can be selected to project an approximate colour RGB image. Texture analysis on this image also produces 75 features per pixel.

This provides 3 different data sets which can be analysed before their results are combined to produce the final result. From each image, 20 mortar and 20 stone unit regions are selected to be used as training data for an SVM classifier. In each case (RGB, hyperspectral and projected RGB), the classification result is filtered first by a vertical line identifying filter and by a horizontal line identifying filter. After morphological post-processing to remove noise, a logical OR operation is used to combine these two 
results into a binary image. This binary image is then combined (using a logical OR) with a marker controlled watershed segmentation of the unprocessed SVM output to produce the final result.

Two flowcharts describing the whole process are shown in Figure 5 and Figure 6 and the results of applying this approach are shown and described in Section 3.2.

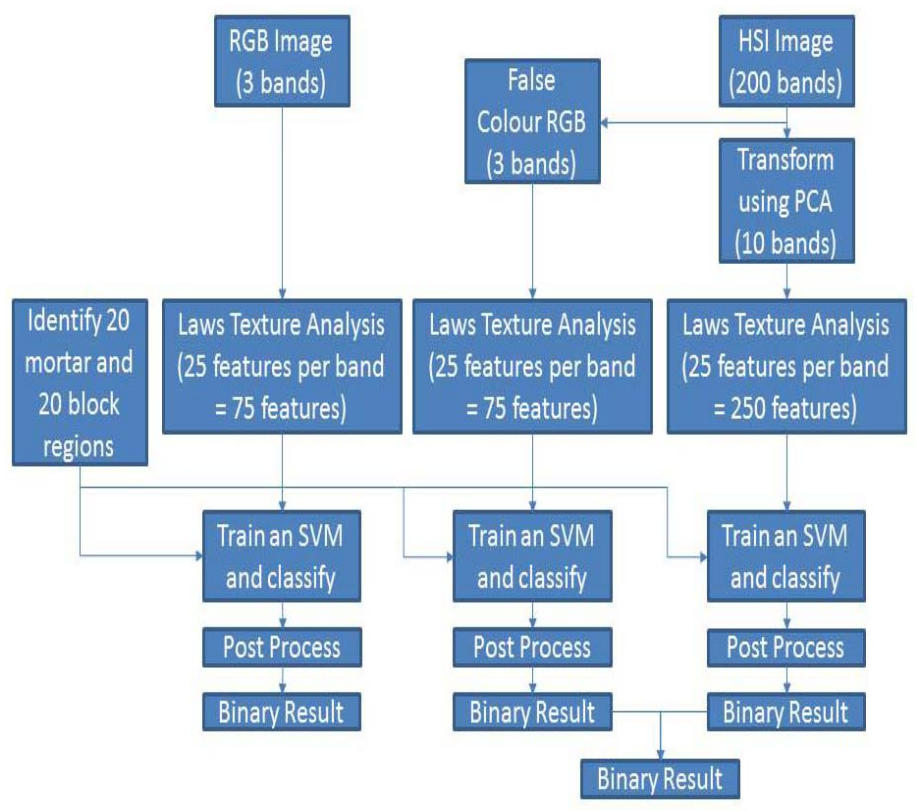

Figure 5: Flowchart describing the mortar detection process.

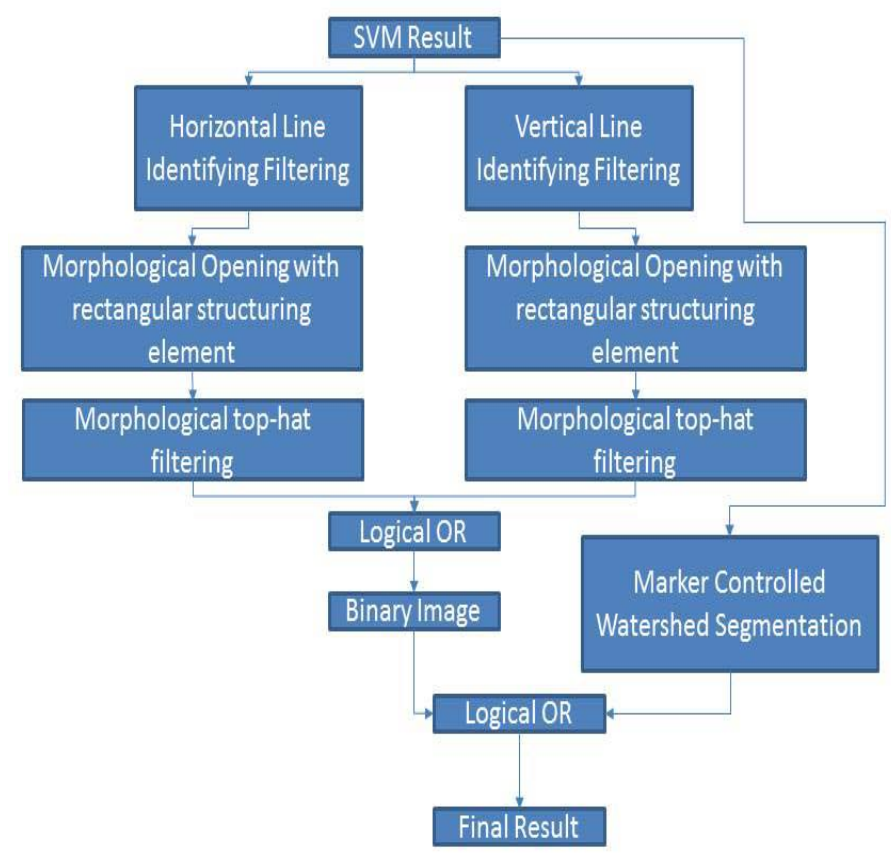

Figure 6: Flowchart describing the post-processing process.

\subsubsection{Pathologies Classification}

As well as identifying mortar, the hyperspectral data can also be used to classify the wall based on the pathologies identified in the manual survey. As with the mortar detection, PCA is used to reduce the data to just 10 bands. This significantly improves the classification speed. An SVM is trained using different class regions which are selected based on the manual survey. Every pixel in the image is then assigned a class by the SVM.

\section{ANALYSIS}

\subsection{Manual survey}

As previously mentioned, the pathologies present in the section of the wall were identified, manually marked and annotated in situ on a scaled elevation drawing. This mark-up drawing was then used to produce a CAD drawing (Figure 7).

Seven different pathologies were identified. The most prevalent was soiling, which is found in sheltered areas exposed to moisture (below string course and to the left hand side of the wall section considered). In some areas, it is also combined with stone exfoliation; some crust has also already been formed.

Other pathologies have also been identified in some stone blocks, such as superficial deposits and swelling. Three stones also have visible cracks which may develop with time into loss of material and structural damage. Table 1 summarises the pathologies identified and the stone units affected.

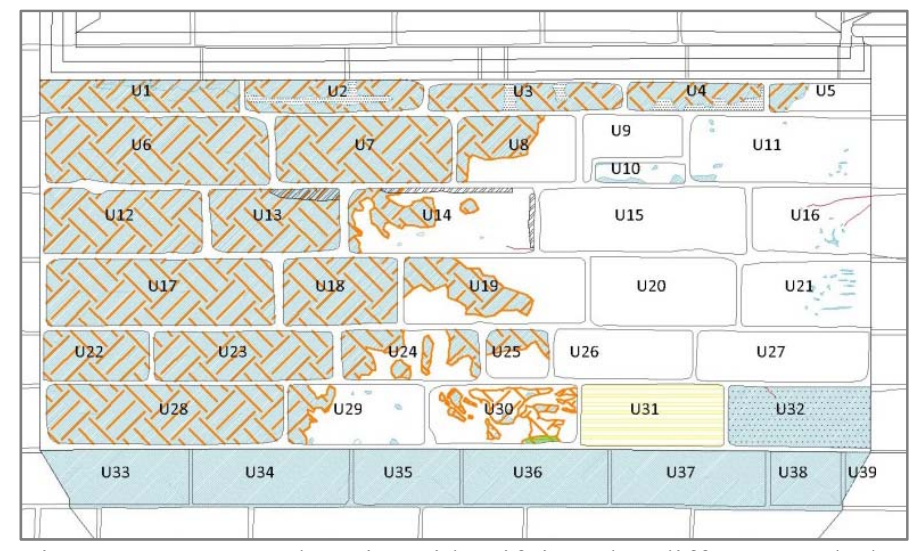

Figure 7: Survey elevation, identifying the different pathologies found on the wall.

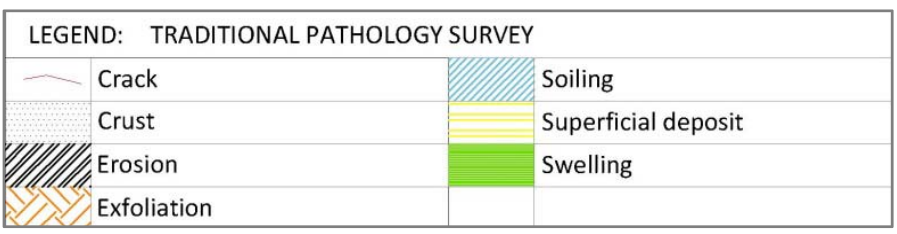

Figure 8: Graphic code used to note pathologies. 


\begin{tabular}{ll}
\hline Pathologies identified & Stone Units affected \\
\hline Crack & U14, U16,U32 \\
Crust & U2-U4, U32 \\
Erosion & U13,U14 \\
Exfoliation & U1-U8, U12-U14, U17-U19, \\
& U22-U25, U28-U30 \\
Soiling & U1-U8, U10-U14, U16-U19, \\
& U21-U25, U28-U30, U32-39 \\
Superficial deposit & U31 \\
Swelling & U30 \\
\hline
\end{tabular}

Table 1: List of identified pathologies and stone units affected.

\subsection{Image Recognition Survey}

\subsubsection{Mortar Detection}

As explained in section 2, three different images were used in total for mortar detection: a standard RGB image, a hyperspectral image and a projected colour RGB image generated from three bands of the hyperspectral image. 20 different mortar regions and 20 different block regions were manually selected to be used as training data for the SVMs. Figure 9 shows the output result of the classifier in each case.

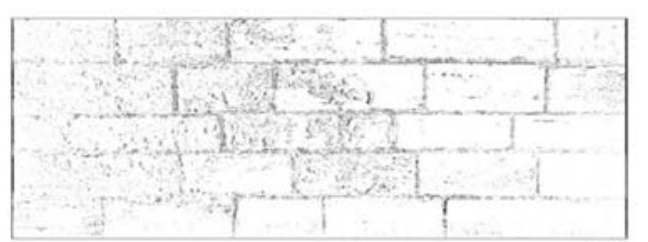

(a)

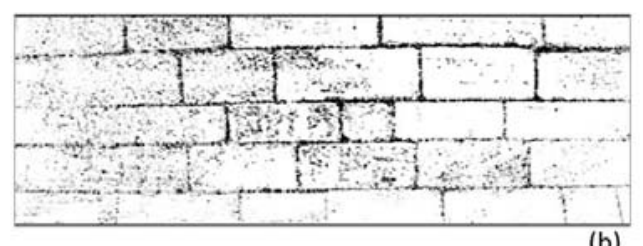

(b)

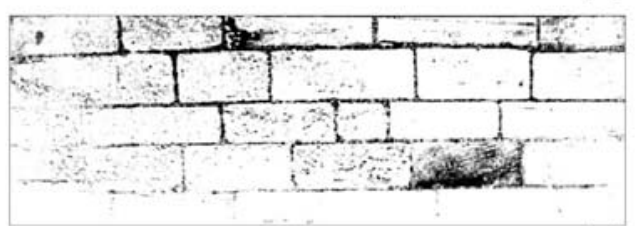

(c)

Figure 9: SVM Mortar Classification Results. (a) RGB, (b) HSI, (c) False Colour RGB.

As can been seen from the SVM mortar classification results, all three images have identified the mortar in different ways. Using the post-processing explained in the methodology section and shown in Figure 6, each of these classification results was used to generate a final binary image where mortar has been detected. These results are shown in Figure 10 (a), (b) and (c). Since (b) and (c) were both generated from the same hyperspectral image, these results were combined using a logical AND function which highlights only regions of mortar that were identified in both results. This is shown in Figure 10 (d).

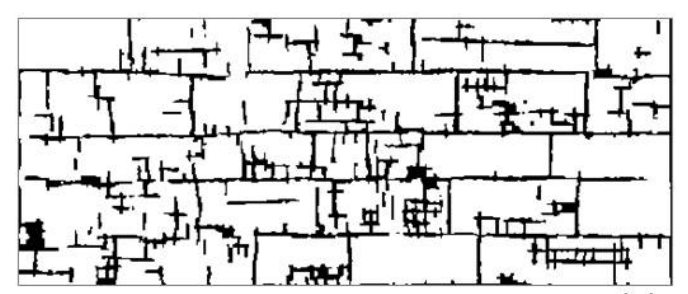

(a)

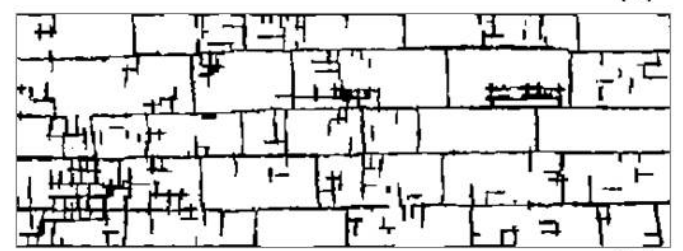

(b)

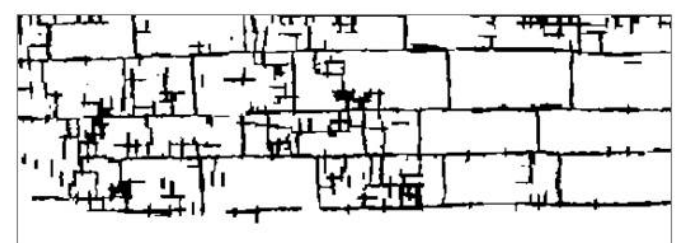

(c)

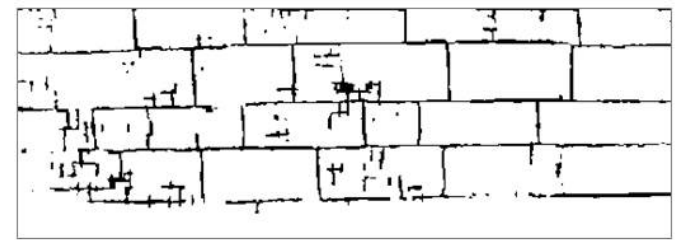

(d)

Figure 10: Post processed mortar classification results (a) RGB (b) Hyperspectral (c) False Colour RGB (d) Combination of hyperspectral and false colour results.

\subsubsection{Pathology Classification}

In the manual survey, 7 different pathologies were identified. However, in many cases, parts of the wall were affected by more than one pathology. For the image processing survey, 5 classes were identified corresponding to different combinations of pathologies. Each of these classes was then assigned a colour. Table 1 shows these classes and colours.

\begin{tabular}{ll}
\hline Class & Colour \\
\hline Exfoliation and Erosion & Green \\
Superficial Deposit & Blue \\
Soiling & Cyan \\
Crust, Soiling and Exfoliation & Red \\
No pathology & Yellow \\
\hline
\end{tabular}

Table 2: Classes and Colours.

Small regions consisting of each of these classes were selected manually. These regions were used as the training data for an SVM classifier and are shown in Figure 11. Since PCA was used, each pixel vector in each training region is made up of 10 values corresponding to the 10 principal components.

The whole image was then classified based on the SVM generated from the training regions. Each pixel was assigned a colour based on the class to which the SVM determined it belonged. This result is shown in Figure 12. 


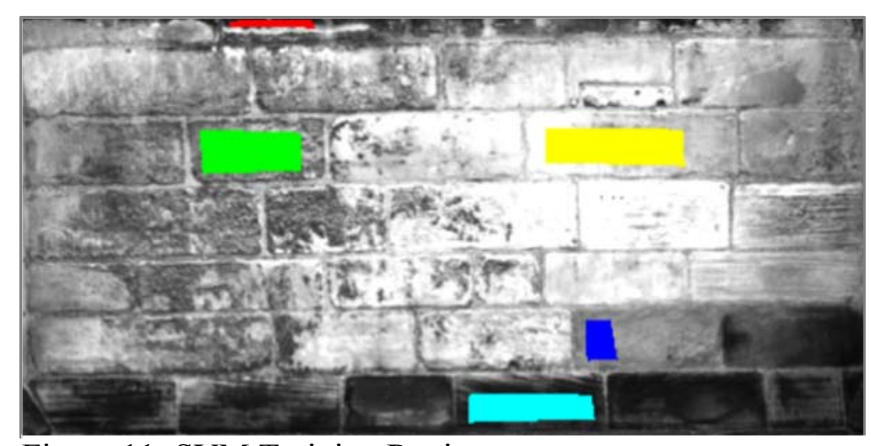

Figure 11: SVM Training Regions.

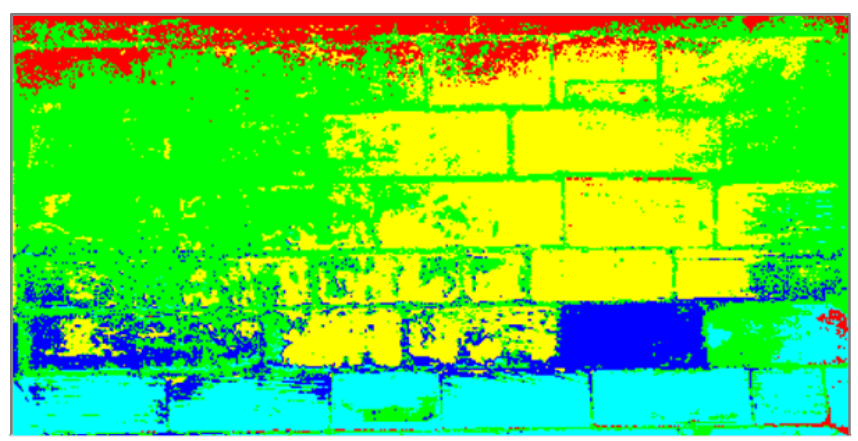

Figure 12: Classification Result.

\section{DISCUSSION}

\subsection{Comparison between the two approaches}

Having presented both the image processing based survey and the traditional survey, this section looks to compare the two methods and evaluates the potential of the image processing based approach as a new methodology for surveying historic sites. To compare the methods, three metrics for comparison were identified: speed, accuracy and site disruption.

\subsubsection{Speed}

The traditional survey in this study took around 2 hours on site with a further 2 hours to draw the results. This does not include the photogrammetry survey of the cathedral provide by Historic Environment Scotland, however it is estimated that for this wall section it would add an additional 4 hours to the process, taking the total time to around 8 hours of work. The data capture required for this image processing survey took around 30 minutes. The algorithms used to detect the mortar regions and classify the pathologies took around 15 minutes to run (on a desktop computer with a $3.6 \mathrm{Ghz}$ Intel Core i7-490 processor running Windows 8 64-bit with 16 GB RAM). Since this was the first trial of the proposed method, several weeks were spent developing the required algorithms. For future surveys, the same algorithms will be used so this process will not need to be repeated meaning the total processing time for a wall of this size could be as little as 45 minutes. Clearly, the image processing based method has great potential to improve the efficiency of the survey process.

\subsubsection{Accuracy}

The traditional approach produces a highly accurate representation of the existing mortar joints and stone units. The results of the mortar detection using the developed image processing approach are representative of the real wall, however, in several cases, some regions were incorrectly classified. See Figure 2, Figure 7 and Figure 10(d). The results of the pathology classification look similar but there are clearly some areas where misclassification has occurred. The stone is not always a homogenous material and exhibits variations in colour and texture and this is where the most of the misclassification occurs. This can be seen by comparing Figure 2, Figure 7 and Figure 12.

\subsubsection{Site Disruption}

For the traditional survey, accessibility has a significant impact on the disruption caused to the building. The wall section surveyed in this paper was at ground-level and so access was not an issue. In other cases, however, scaffolding is often required to allow the expert to get close enough for inspection. This can cause temporary closure of the building and a significant increase in costs. With the image processing based approach the disruption to the building can be minimised as general access to the area only needs to be restricted during imaging. In cases where scaffolding would be required for a manual survey, drones equipped with cameras and other image sensors could be used to carry out the data capture.

\subsection{Future Work}

\subsubsection{Mortar Detection}

So far, the mortar detection results have only been used to produce a drawing highlighting where the system detected mortar. From these results, however, it is expected that further analysis could automatically determine the thickness of the joints and the size and geometry of the masonry units. At this stage, the results are just images. In further work, these images can be transformed into CAD drawings by establishing a relationship between the pixels of the image and the $(\mathrm{x}, \mathrm{y}, \mathrm{z})$ coordinates in CAD space.

\subsubsection{Pathology Classification}

In this study, the training regions were selected from the same image that was classified. In future, a comprehensive spectral library of pathology classes can be developed and maintained. This library will re- 
duce the need for training data to be selected for future surveys allowing the analysis of a much wider range of stone masonry walls.

\section{CONCLUSIONS}

In this paper, an alternative method of surveying historic buildings using image processing techniques is proposed. Both RGB and hyperspectral images were captured and processed in order to identify mortar joints and to classify different pathologies. A comparison with the traditional method shows promising results. In terms of speed and site disruption, a number of significant improvements are offered. At this stage, the mortar detection and pathology classification results provide only an estimate of the condition of the wall. To reach the level of accuracy required for a complete architectural conservation condition survey, this estimate must be improved. With this in mind, a number of areas for further development were identified giving the project clear scope for future research.

\section{ACKNOWLEDGEMENTS}

The authors wish to acknowledge Historic Environment Scotland (HES) for giving permission to survey the Glasgow Cathedral and providing them with the CAD files resulting from the photogrammetry survey and 3D laser scanner point cloud as well as site induction and assistance while carrying out the works.

\section{REFERENCES}

Beucher, S. 1991 'The Watershed Transform Applied to Image Segmentation' Proceedings of the Pfefferkorn Conference on Signal and Image Processing in Microscopy \& Microanalysis,pp.299-314

Bulanona, D.M., Kataokab, T., Otac, Y. \& Hiromac, T. 2002, 'Automation and Emerging Technologies: A Segmentation Algorithm for the Automatic Recognition of Fuji Apples at Harvest', Journal of Agricultural Engineering Research, vol.83, no4, pp.405-412.

Cortes, C. \& Vapnik, V. 1995 'Support-vector networks' Machine Learning,Vol.20,no3,pp.273-297

Dougherty, G. 2009 Digital Image Processing for Medical Applications, Cambridge Univ. Press, NY

Colloquium 1990, EE Colloquium on the Role of Image Processing in Defence \& Military Electronics

Hemmleb, M., Weritz, F. \& Maierhofer, C. 2005 'Damage detection on buildings surfaces with multispectral techniques' CIPA 2005 XX International Symposium, Torino, Italy.

Hemmleb, M., Weritz, F., Schiemenz, A., Grote, A. \& Maierhofer, C. 2006 'Multi-spectral data ac- quisition and processing techniques for damage detection on building surfaces' Image engineering and vision metrology, Dresden, Germany.

Jollie, I.T 2002 Principal Component Analysis, Springer Series in Statistics, Springer.

Kelman, T., Ren, J. \& Marshall, S. 2013 'Effective classification of Chinese tea samples in hyperspectral imaging' Sciedu Press, Artificial Intelligence Research, vol. 2, no. 4

Lerma, J.L. 2001 'Multiband versus multispectral supervised classification of architectural images' The photogrammetric record, vol.17,no97,pp.89-101

Laws, K.I. 1980 'Rapid Texture Identification'. Proc. SPIE 0238, Image Processing for Missile Guidance, 376.

Lerma, J.L. 2005 'Automatic plotting of architectural facades with multispectral images', Journal of surveying engineering, vol.131, no3, pp.73-77

Lerma, J.L., Ruiz, L. \& Buchon, F., 2000 'Application of spectral and textural classifications to recognize materials and damages on historic building facades' International Archives of Photogrammetry and Remote Sensing, Amsterdam, vol.XXXIII, Part B5, pp480-484

Palombi, L., Lognoli, D., Raimondi, V., Cecchi, G., Hällström, J., Barup, K., Conti, C., Grönlund, R., Johansson, A. \& Svanberg, S. 2008 'Hyperspectral fluorescence lidar imaging at the Colosseum, Rome: Elucidating past conservation interventions', Optics express, vol.16, no10, pp.6794-6808.

Qiao, T., Ren, J., Craigie, C., Zabalza, J., Maltin, Ch. \& Marshall, S. 2015 'Quantitative Prediction of Beef Quality Using Visible and NIR Spectroscopy with Large Data Samples Under Industry Conditions', Journal of Applied Spectroscopy, vol.82, no1, pp.137-144

Serra, J. 1988 Image Analysis and Mathematical Morphology, Volume 2: Theoretical Advances, Academic Press, Inc. Orlando, FL, USA

Sewaga, E., Miura, M. \& Abe, D. 2006 'Image Recognition Wide-Area Surveillance Technology for Safety and Security in Society' FUJITSU Scientific \& Technical Journal, vol.43, no2, pp.204-211

Sidiropoulou-Velidou, D., Georgopoulos, A. \& Lerma,J.L. 2012 'Exploitation of thermal imagery for the detection of pathologies in monuments' Lecture Notes in Computer Science, vol.7616,pp.97-108

Zabalza, J., Ren, J., Ren, J., Liu, Z. \& Marshall, S. 2014 'Structured covariance principal component analysis for real-time onsite feature extraction and dimensionality reduction in hyperspectral imaging' OSA Applied Optics, vol.53, no20, pp.4440-4449

Zabalza, J., Ren, J., Zheng, J., Han, J., Zhao, H., Li, S. \& Marshall, S 2015 'Novel two dimensional singular spectrum analysis for effective feature extraction and data classification in hyperspectral imaging' IEEE Transactions on Geoscience and Remote Sensing, vol. 53, no. 8, pp.4418-4433 